\title{
The Cimicifuga preparation BNO 1055 vs. conjugated estrogens in a double-blind placebo-controlled study: effects on menopause symptoms and bone markers
}

\author{
W. Wuttke ${ }^{\mathrm{a}, *}$, D. Seidlová-Wuttke ${ }^{\mathrm{a}}$, C. Gorkow ${ }^{\mathrm{b}}$ \\ a Department of Clinical and Experimental Endocrinology, University of Göttingen, Robert-Koch-Strasse 40, 37075 Göttingen, Germany \\ ${ }^{\mathrm{b}}$ Bionorica $A G$, Neumarkt, Germany
}

Received 20 May 2002

\begin{abstract}
Objectives: In the present study, therapeutic effects of the Cimicifuga racemosa preparation CR BNO 1055 (Klimadynon ${ }^{\circledR} /$ Menofem ${ }^{\circledR}$ ) on climacteric complaints, bone metabolism and endometrium will be compared with those of conjugated estrogens (CE) and placebo. The question whether CR BNO 1055 contains substances with selective estrogen receptor modulator (SERM) activity will be investigated. Methods: Sixty-two evaluable postmenopausal women were included in the double-blind, randomized, multicentre study, and treated either with CR BNO 1055 (daily dose corresponding to $40 \mathrm{mg}$ herbal drug), $0.6 \mathrm{mg} \mathrm{CE}$, or matching placebo, for 3 months. Menopausal symptoms were assessed by the menopause rating scale (MRS) and a diary. Levels of CrossLaps (marker of bone degradation) were determined by ELECSYS system and bone-specific alkaline phosphatase (marker of bone formation) by an enzymatic assay. Endometrial thickness was measured via transvaginal ultrasound; vaginal cytology was also studied. The primary efficacy criterion was the change from baseline to end point in the MRS. Change from baseline was analyzed for the secondary variables too. Results: CR BNO 1055 proved to be equipotent to CE and superior to placebo in reducing climacteric complaints. Under both preparations, beneficial effects on bone metabolism have been observed in the serum. CR BNO 1055 had no effect on endometrial thickness, which was significantly increased by CE. Vaginal superficial cells were increased under CE and CR BNO 1055 treatment. Conclusion: The results concerning climacteric complaints and on bone metabolism indicate an equipotent effect of CR BNO 1055 in comparison to $0.6 \mathrm{mg}$ CE per day. It is proposed that CR BNO 1055 contains substances with SERM activity, i.e. with desired effects in the brain/ hypothalamus, in the bone and in the vagina, but without exerting uterotrophic effects.
\end{abstract}

(C) 2003 Elsevier Science Ireland Ltd. All rights reserved.

Keywords: Phytoestrogens; Menopausal complaints; Cimicifuga racemosa; Bone metabolism; SERM

\section{Introduction}

* Corresponding author. Tel.: +49-551-396714; fax: +49551-396518.

E-mail address: ufkendo@med.uni-goettingen.de (W. Wuttke).
Phytoestrogens are currently in the focus of interest, since it is known that classical estrogen/ progestin replacement therapy (HRT) significantly 
increases the risk of mammary cancer [1-3]. Recently, the American National Institute of Health stopped a clinical trial with HRT in healthy postmenopausal women due to an increased risk of invasive breast cancer and an increase of cardiovascular risks including venous thrombosis. These risks outweigh any benefits of HRT [3]. An estrogen replacement therapy also increases the risk of endometrial cancer [4], which is reduced by additional progestin administration [5]. The strong belief that HRT will reduce the risk for cardiovascular diseases [6,7] has been challenged by the finding of increased fatal heart attacks in women with pre-existing arteriosclerosis $[8,9]$. Experimental and clinical investigations about the effects of phytoestrogens on the cardiovascular system show inconsistent results [10,11]. As a consequence, the compliance of classical hormone therapies decreases and many patients look for alternatives. General and industrial interest on the effects of phytoestrogens have been focussed on soy- or redclover-derived isoflavones and on extracts of black cohosh (Cimicifuga racemosa or CR).

In postmenopausal women, $17 \beta$-estradiol $\left(\mathrm{E}_{2}\right)$ and conjugated estrogens (CE) have a desired antiosteoporotic effect. Under HRT, bone mineral density (BMD) can be maintained, almost stable, as long as women practice HRT [12]. Osteoprotective effects of CR extracts are supposed, but not yet proved.

The vagina of postmenopausal women suffers often from ascending infections because the estrogen-enforced acid milieu is missing. Furthermore, vaginal dryness often impairs sexual desires within partnerships [13].

A serious side effect of the progestin-unopposed estrogen treatment is the stimulation of the endometrium, which leads to an increased risk of endometrial cancer [4]. Progestin application opposes this effect [5], but often leads to uterine bleedings. Thus, the compliance of patients for HRT decreases. Experiments in rodents as well as clinical studies in postmenopausal women demonstrated that $\mathrm{CR}$ extracts do not have uterotrophic effects $[14,15]$, which was the basis for the assumption that they do not contain estrogenic compounds [16]. Yet, in ovariectomized rats, the aqueous/ethanolic extract BNO 1055 exerted es- trogenic effects in the hypothalamus, in the bone and in the vagina (Wuttke et al., and SeidlováWuttke et al., this volume). With the increasing knowledge of the organ-selective action of selective estrogen receptor modulators (SERMs) [17], it seems necessary to re-evaluate possible estrogenic actions of $\mathrm{CR}$ extracts also in postmenopausal women.

The synthetic SERM raloxifene was shown not to have uterine effects, but to exert estrogenic effects in bones and on lipid metabolism [18]. The question arises, whether CR BNO 1055 contains substances exerting SERM activity, i.e. no undesired effects in the uterus, but desired effects on the GnRH pulse generator, on the bones and in the vagina.

The aim of this study was to examine, whether CR BNO 1055 (Klimadynon ${ }^{\circledR} /$ Menofem ${ }^{\circledR}$ ), in comparison to a standard hormone therapy and placebo, improves climacteric complaints and has positive estrogenic effects in the vagina and on bone metabolism, without showing uterotrophic activity.

\section{Materials and methods}

\subsection{Study design}

This double-blind, randomized, placebo- and CE-controlled multicentre study, in parallel groups, was conducted in accordance with the good clinical practice (GCP) guidelines [19], the declaration of Helsinki [20] and the regulations of independent ethics commissions. Quality was assured by monitoring according to standard operating procedures (SOPs) and by independent audits (one in-house audit at Sponsor and CRO, three out-side audits).

To guarantee the balance of three different treatments per trial centre, patients were randomized to treatments using a randomly permuted block design [21].

Data were collected at week 0 (baseline) and at weeks 4,8 and 12 under treatment. Data management was conducted according to GCP guidelines. 


\subsection{Inclusion criteria}

Patients meeting the following inclusion criteria were eligible for enrollment:

Postmenopausal women, 40-60 years of age, body mass index $(\mathrm{BMI})<30$, last menstrual bleeding at least 6 months ago, i.e. perimenopausal women with postmenopausal hormone values $\quad(17 \beta$-estradiol $\leq 40 \mathrm{pg} / \mathrm{ml}, \quad \mathrm{FSH} \geq 25$ $\mathrm{mU} / \mathrm{ml}$ ) at all visits, at least three hot flushes per day (as documented in a diary), menopause rating scale (MRS) [22] items 1-6, sum of scores $\geq 1.7$ at visits 1 and 2 , MRS item 1 (hot flushes) $\geq 0.3$ at visits 1 and 2 .

Initially, a total of 97 patients were randomized (intention to treat = ITT-collective). Two patients dropped out prematurely, due to the withdrawal of informed consent after baseline. Post-baseline values for these two women did not exist.

The ITT analyses did not reveal significant differences between the treatment groups. In the course of the study, some patients had

- masked ovulatory or unovulatory cycles $\left(\mathrm{E}_{2}\right.$ levels $>50 \mathrm{pg} / \mathrm{ml}$, FSH levels $<25 \mathrm{mU} / \mathrm{ml}$, or LH levels $<12 \mathrm{mU} / \mathrm{ml}$ ) and

- relevant estrogen production in the fatty tissue $\left(\right.$ BMI $\left.>30 \mathrm{~kg} / \mathrm{m}^{2}\right)$.

Therefore, 33 perimenopausal patients were excluded from the statistical analysis of the ITT collective. These patients were defined as protocol violators, who could interfere with the results, e.g. could show false-positive results.

Despite the exclusion of 33 patients, the allocation of the true postmenopausal women to the treatments was well balanced (CR BNO 1055: $n=$ 20; CE: $n=22$; placebo: $n=20$ ). Hence, the protocol correct collective consisted of $n=62$ patients. At the start of the treatment, patient characteristics concerning age, height, weight, intensity of climacteric symptoms, etc. were comparable in all treatment groups. An analysis of pillcount data demonstrated a high level of compliance in all the three treatment groups.
After receiving a written and verbal information about type, purpose, risks, benefits, duration of the study and information about alternative therapies, all included patients had to sign a written informed consent prior to any studyrelated measurements.

\subsection{Study drugs}

All three trial medications (CR BNO 1055 (Klimadynon ${ }^{\circledR} /$ Menofem $^{\circledR}$ ), corresponding to 40 $\mathrm{mg}$ herbal drug per day; CE, $0.6 \mathrm{mg}$ per day; placebo) consisted of hard gelatin capsules with magnesium stearate and lactose as bulk excipients.

One group received a preparation of dried aqueous/ethanolic $(58 \%, \mathrm{v} / \mathrm{v})$ extract of the rhizome of Cimicifunga racemosa (CR BNO 1055). Each capsule (Batch No. 805005) contained extract corresponding to $20 \mathrm{mg}$ herbal drug in the form of one film-coated tablet of Klimadynon ${ }^{\circledR /}$ Menofem ${ }^{\circledR}$ (grinded). The daily dose was two capsules.

One group received a daily dose of two capsules with $0.3 \mathrm{mg}$ CE per capsule. Each capsule (Batch No. 71011210) consisted of one Oestrofeminal ${ }^{\circledR}$ capsule (content per capsule: oestron $75-85 \%$, equilin $6-15 \%$, equilenin up to $4 \%, 17 \alpha$-estradiol, $17 \alpha$-dihydro-equilin, $17 \alpha$-dihydro-equilenin $2-8 \%$ ) of Heinrich Mack Nachf., Germany. One group received matching placebo capsules (Batch No. 805001). The daily dose was two capsules.

After the run-in-period of 2 weeks, patients started with study medication on day 0 (visit 2, baseline). For this purpose, eligible patients were randomized and provided with study medication. Appropriately packed and labeled individual containers with 70 capsules were disposed at visit 2 (for weeks 1-4), visit 3 (for weeks 5-8), and at visit 4 (for weeks 9-12).

All three preparations were identical in appearance. The study medications were produced according to the standards of good manufacturing practice (GMP). Certificates of analysis before and during the study and descriptions of the investigational products were filed with the study documentation. 


\subsection{Efficacy/tolerability}

MRS [22] was chosen as the primary efficacy criterion. The questionnaire (Fig. 1) was filled in by the patients at baseline (week 0 ) and after 4,8 , and 12 weeks.

According to a factor analytical study [23], MRS items are not independent and can be combined. It was demonstrated that the MRS items loaded on three main factors. For further statistical analyses of the present study, we adopted a three-factorial solution as a linear combination of MRS items. In this three-factorial system, the MRS items 1-3 (hot flushes/sweating, heart complaints, sleep disorders) load on factor 1 "hot flushes". The items 4-6 (depressive moods, nervousness/irritability, impaired performance/ memory) load on factor 2 "psyche". The items 7-10 (disorders of sexuality, urinary symptoms, vaginal dryness, joint and muscle symptoms) load on factor 3 "atrophy".

During the run-in-period of 2 weeks and during the entire study, patients had to fill out a diary concerning daily number of hot flushes, the occurrence (intensity, duration) of vaginal bleeding episodes and sleep disturbances (frequency of awaking per night, nights waking up early, nights with problem to fall asleep).

At week 0 (baseline) and after 4, 8, and 12 weeks, blood samples were collected for routine safety laboratory parameters and for determination of $\mathrm{LH}, \mathrm{FSH}$, estradiol and progesterone. Additionally, the levels of CrossLaps (marker for bone degradation) were determined by immunoassays (Hormones and CrossLaps were measured with the ELECSYS System, Roche-Diagnostics, Mannheim) and the levels of bone-specific alkaline phosphatase (marker for bone formation) by an enzymatic assay (Hitachi/Roche-Diagnostics, Mannheim).

At baseline (week 0) and after 12 weeks, all patients had a gynecological investigation, which included transvaginal ultrasound for determination of the endometrial thickness and vaginal smear [24] to determine the maturity index of vaginal epithelium, i.e. estrogenic activity of the study medications.

\title{
Menopause Rating Scale
}

\section{Explanation of the menopausal symptoms}

\author{
1. Ascending feeling of heat, outbreaks of sweating (frequency, severity) \\ 2. Palpitations, racing heart, galloping heart, feeling of being stifled \\ 3. Difficulty falling asleep, difficulty staying asleep, waking up too early \\ 4. Despondency, sadness, weepiness, lack of drive, modd swings \\ 5. Nervousness, inner tension, aggressiveness \\ 6. Physical and mental fatigue, difficulty concentrating, forgetfulness \\ 7. Decrease in sexual desire, sexual activity and satisfaction \\ 8. Complaints on urination, frequenture to urinate, involuntary urination \\ 9. Feeling of dryness in the vagina, difficulties with sexual intercourse \\ 10. Pain mainly in the finger joints, rheumatic-like pains, tingling
}

Please answer all ten questions by crossing the vertical line as in the example below!

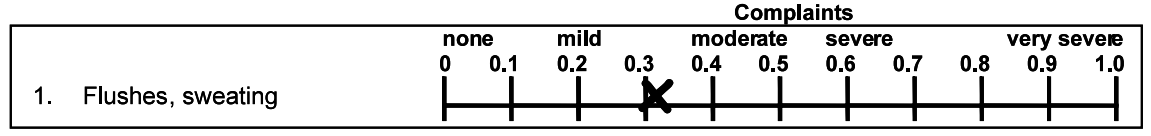

Fig. 1. MRS questionnaire [22]. 
At all visits, patients were asked about the occurrence of adverse events (type, severity, duration) and about new medications or changes of pre-existing concomitant medications.

\subsection{Statistical analyses}

The primary efficacy criterion was the change from baseline to end point after 12 weeks of treatment in MRS (items 1-10).

Secondary variables were also analyzed, with respect to the change from baseline to end point, after 12 weeks of treatment. The occurrence of serious and non-serious events and the results of the vaginal cytology were presented as listings or change of proportional cell counts, respectively.

For each variable, multiple comparisons of the treatment groups were performed by analysis of covariance. Thereby, point estimators for the baseline and center-adjusted mean values as well as the confidence intervals and $P$-values were calculated. Statistical analyses were accomplished with SAS.

\section{Endometrial Thickness}

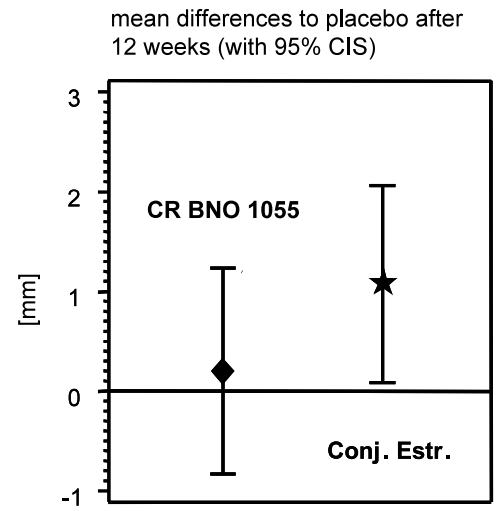

Fig. 2. Endometrial thickness was significantly increased by the $\mathrm{CE}$, but not by $C$. racemosa BNO 1055 . Shown are the mean differences to placebo after 12 weeks with the $95 \%$ confidence intervals (CI). The lacking overlap of CI with upper value of placebo-treated controls indicates that the effects of the $\mathrm{CE}$ were statistically significant.

\section{Results}

Fig. 2 details the behaviour of endometrial thickness from a pre-treatment baseline until the end of the treatment in the three groups of patients. Under placebo, the median endometrial thickness remained unchanged over the treatment period. Fig. 2 shows the mean differences to placebo after the 12-week treatment with the 95\% confidence intervals. Under CR BNO 1055, the median endometrial thickness remained unchanged. The median increase under CE was more than $1 \mathrm{~mm}$ which was statistically significant in comparison to placebo. The $\mathrm{CE}$ had also a significant effect on the amount of superficial cells in the vaginal smear. Vaginal superficial cells were also slightly stimulated by Klimadynon ${ }^{\circledR}$, while their number was decreased in the placebo group (Fig. 3).

Analysis of the total scores over all 10 MRS items showed that CR BNO 1055 (Klimadynon ${ }^{\circledR} /$ Menofem ${ }^{\circledR}$ ) was as effective as CE in reducing climacteric complaints, when compared with the placebo group (Fig. 4). In Fig. 4 and all the following figures, changes from baseline values are displayed as mean values for all patients of the protocol correct collective. Increases or decreases from baseline values during treatment are displayed above or below zero, respectively. They are given in the appropriate dimension. Fig. 5 shows the effects of the treatment on the three factors. In comparison to placebo, the MRS items, which

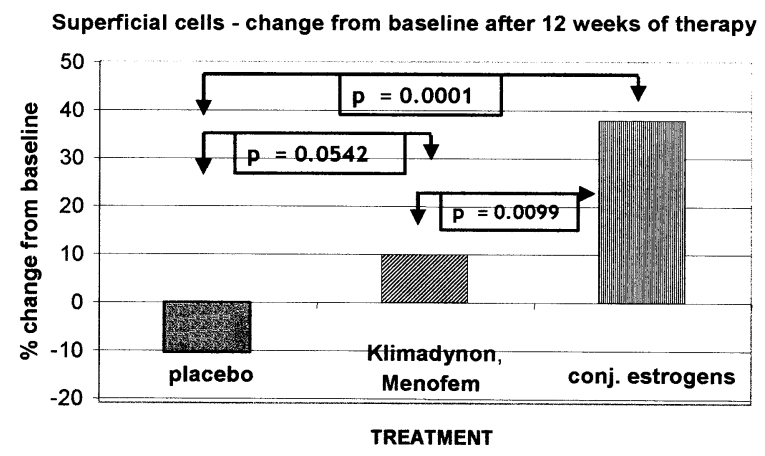

Fig. 3. In contrast to placebo, under C. racemosa BNO 1055, a slight increase of superficial cells was observed and this effect approached significance. CE significantly increased the number of superficial cells. 
Menopause Rating scale (MRS): Items 1-10

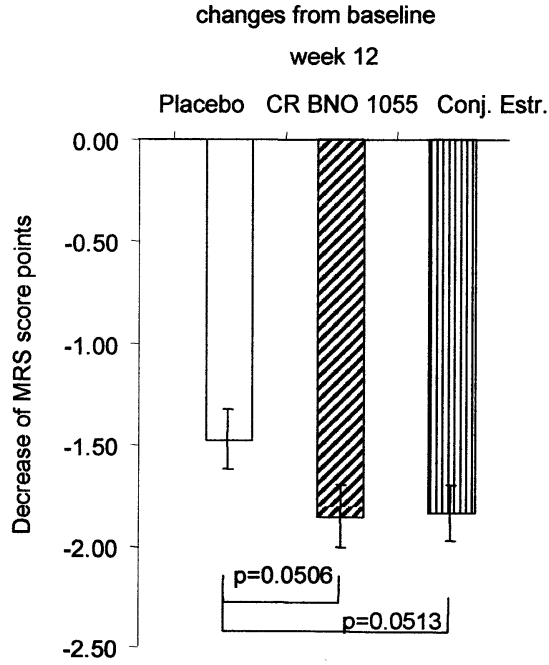

Means adjusted for baseline and center differences +/- SEM

Fig. 4. Evaluation of MRS (i.e. items 1-10) demonstrates an obvious placebo effect over the 12-week treatment period, which was outmatched by CR BNO 1055 (daily dose; corresponding to $40 \mathrm{mg}$ herbal drug) and CE. These effects approached significance.

load on factor 1 "hot flushes" (MRS items 1-3) were significantly reduced by the CE. CR BNO 1055 showed a marked difference to placebo, without reaching the level of significance. Similarly, the climacteric complaints loading on factor 2 "psyche" (MRS items 4-6) were distinctly improved by CR BNO 1055. The effect of the $\mathrm{CE}$ was less obvious. The analysis of the factor 3 "atrophy" (MRS items 7-10) revealed a significant decrease under CR BNO 1055 whereas the difference under $\mathrm{CE}$, in comparison to placebo, did not reach the level of significance.

From the evaluation of the diary, positive effects on the frequency of waking up during the night at week 12 and waking up early, at weeks 8 and 12 have been observed under treatment with $\mathrm{CR}$ BNO 1055, in comparison to placebo (data not shown).

Analyses of markers of bone metabolism in the serum of postmenopausal women indicated that CR BNO 1055 and CE had comparable beneficial effects. CrossLaps are the metabolic products of bone-specific collagen- $1 \alpha 1$ and therefore generally accepted as markers of bone degradation. They increased in the placebo group, decreased under $\mathrm{CR} \mathrm{BNO}$ and decreased under $\mathrm{CE}$ at week 12 (Fig. 6). This indicates decreased activity of the osteoclast cells, which are responsible for bone degradation.

The metabolic marker for bone formation, bone-specific alkaline phosphatase, remained unchanged in the placebo group, significantly increased under CR BNO 1055 at week 12, and remained unchanged under $\mathrm{CE}$ (Fig. 7). This indicates an increased activity of the osteoblast cells, which are responsible for bone formation, under C. racemosa BNO 1055.

CR BNO 1055 seems to increase osteoblast activity, while it slightly decreases osteoclast activity. This is demonstrated by an index, which describes the effects on bone turnover. The index, $\log$ (bone alkaline phosphatase: CrossLaps), showed significant increases for CR BNO 1055 and $\mathrm{CE}$ in comparison to placebo (Fig. 8).

CR BNO 1055 and $\mathrm{CE}$ tended to increase triglycerides. None of the other evaluated routine safety parameters showed clinically relevant changes, which could be interpreted as systematic treatment effects. The incidence of non-serious adverse events of mild to moderate severity were comparable in the three treatment groups. No serious adverse event occurred.

\section{Discussion}

In this study, it was the first time that the therapeutic effects of an aqueous/ethanolic extract, C. racemosa BNO 1055 (Klimadynon ${ }^{\circledR} /$ Menofem ${ }^{\circledR}$ ), were investigated in a randomized three-armed, double-blind, and GCP-conform clinical trial. The comparison included a placebo and a positive control in the form of CE. The results clearly indicate that CR BNO 1055 has desired SERM activities, not only in the rat (Wuttke et al., this volume; Seidlová-Wuttke et al., this volume) but also in postmenopausal women. While CE significantly increased endometrial thickness of the postmenopausal women, such effect was not seen in the CR BNO 1055 treated patients. It was repeatedly shown in the past that 


\title{
Menopause Rating Scale: Factor Analysis
}

\author{
changes from baseline
}

week 12

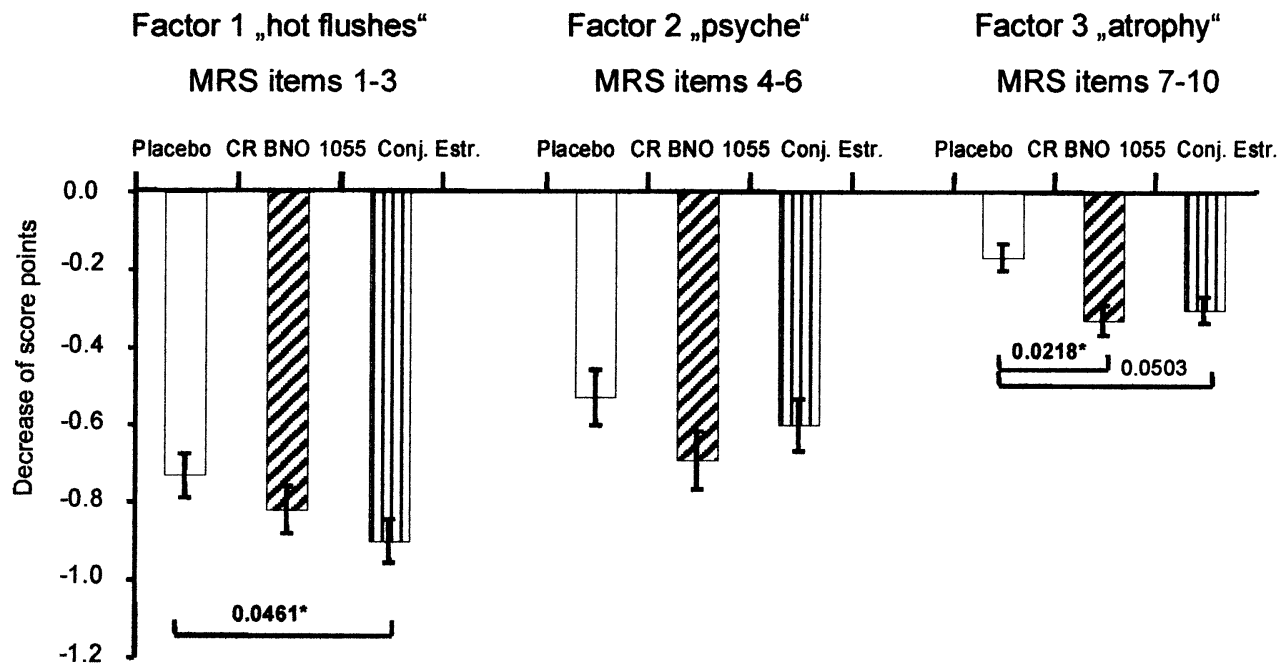

Means adjusted for baseline and center differences $+/$ - SEM

Fig. 5. According to a factor analysis, the 10 items of the MRS were loaded on three factors [23]. Factor 1 "hot flushes" was obviously improved by placebo and significantly improved under CE. Under CR BNO 1055, this effect was similar, but statistically not significant. Factor 2 "psyche" was improved by placebo and further improved by the CE and CR BNO 1055. These effects were statistically not significant. Factor 3 "atrophy" was slightly influenced by placebo. A significant improvement was obtained under CR BNO 1055, while the CE approached significance.

CR extracts do not stimulate uterine weight [1315] and the expression of estrogen-regulated uterine genes and their protein products. $17 \beta$-Estradiol increased these parameters (Wuttke et al., this volume; Seidlová-Wuttke et al., this volume). Hence, it appears safe to conclude that the substance(s) with SERM activity present in CR BNO 1055 do not address the uterus. It is, therefore, unlikely that they may stimulate the endometrium to such an extent that an endometrial cancer may develop. Consequently, a treatment with CR BNO 1055 can be performed without additional progestin application.

In the vagina, estrogens exert desired effects by stimulating acidity of the vaginal milieu. A lower $\mathrm{pH}$ prevents ascending infections [25]. The increased number of superficial cells in the vaginal smears of patients treated with $\mathrm{CE}$ is a clear sign for estrogenic effects in the vagina. In comparison to placebo, vaginal superficial cells were also increased under CR BNO 1055 and this effect approached significance. Not only lowering of the vaginal $\mathrm{pH}$ but also lubrication upon sexual arousal is a desired estrogenic effect. Hence, CR BNO 1055 may fulfill in part also these desired criteria by exerting a mild estrogenic effect in the vagina of postmenopausal women.

In a previous placebo-controlled study, using a higher than recommended CR doses [25] as well as in the present investigation, climacteric complaints were significantly reduced by treatment with placebo preparations. In addition to this placebo effect, both, CR BNO 1055 and the CE, had in the present study favorable and significant effects on climacteric complaints. We utilized the MRS [22] to estimate the effects of the treatment on climacteric complaints. Additionally, we followed the proposal to subcategorize the $10 \mathrm{MRS}$ items into three factors [23]. The factor 1 "hot flushes" comprises the MRS item 1 "hot flushes", item 2 


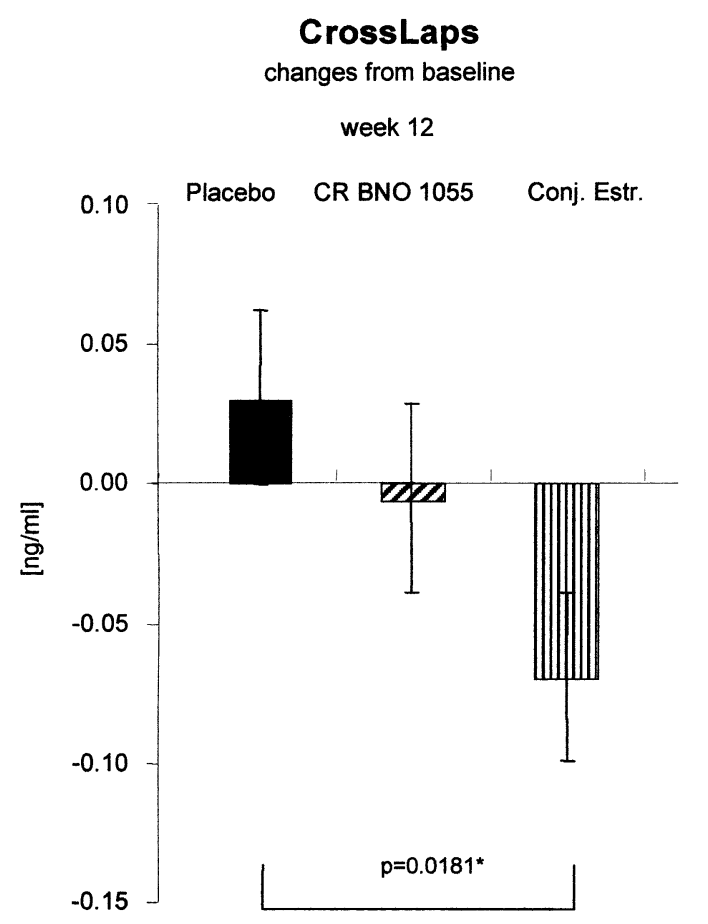

Means adjusted for baseline and center differences +/- SEM

Fig. 6. Serum levels of CrossLaps were significantly reduced by $\mathrm{CE}$, an effect not seen under C. racemosa BNO 1055. In contrast to placebo, CrossLaps did not show an increase under CR BNO 1055.

"cardiac symptoms", and item 3 "sleep disorders". This factor was obviously reduced by placebo and a significant improvement was noted under CE. CR BNO 1055 also showed an improvement, which, however, was not statistically significant. The factor 2 "psyche" comprises the MRS item 4 "depressive mood", item 5 "nervousness"/"irritability" and item 6 "impaired performance/memory". Factor 2 was obviously improved by placebo. A stronger improvement, though statistically not significant, was observed under CR BNO 1055 and CE. The factor 3 "atrophy" comprises the MRS item 7 "disorders of sexuality", item 8 "urinary symptoms", item 9 "vaginal dryness", and item 10 "joint and muscle symptoms". This factor was least improved under placebo, but significantly improved under CR BNO 1055 and CE.

\section{Bone - Specific Alkaline Phosphatase}

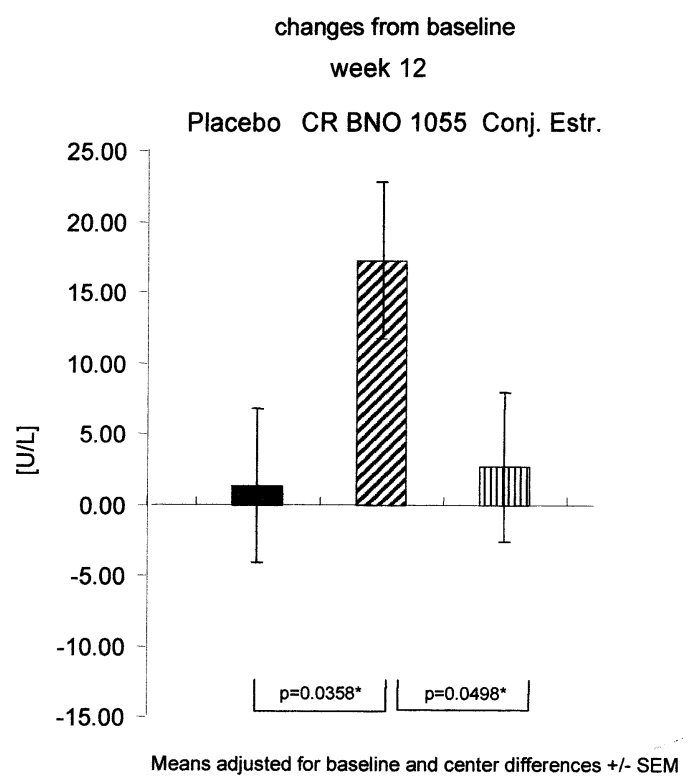

Fig. 7. Serum levels of bone-specific alkaline phosphatase were significantly increased after 12 weeks of treatment with $C$. racemosa BNO 1055, an effect not seen under CE.

This study showed that treatment with placebo reduces the intensity of climacteric complaints. Particularly, susceptive to this placebo effect is the factor 1 "hot flushes". Less susceptive is the factor 2 "psyche" and least susceptive is the factor 3 "atrophy". The data also demonstrate that both, CE as well as CR BNO 1055, improved the three MRS factors in addition to a placebo effect. Analysis of the total MRS Score showed that CR BNO 1055 was as effective as CE in reducing climacteric complaints, when compared with placebo.

The effects of CR BNO 1055 on neurovegetative symptoms are undoubtedly exerted in the brain. Items like hot flushes and tachycardiac episodes emerge due to an overactivation of the hypothalamic GnRH pulse generator, in consequence to a deficiency of estrogens [26]. In human, the overactivation was shown by a simultaneous occurrence of hot flushes and $\mathrm{LH}$ pulses in the serum. This effect is exerted by hypothalamic mechanisms, a structure where the GnRH pulse generator resides in [27]. Investigations with rats repeatedly showed a reduction of $\mathrm{LH}$ pulsatility under aqu- 
Bone Turnover Index

log (Bone-spec. Alk. Phoshatase / CrossLaps)

changes from baseline

week 12

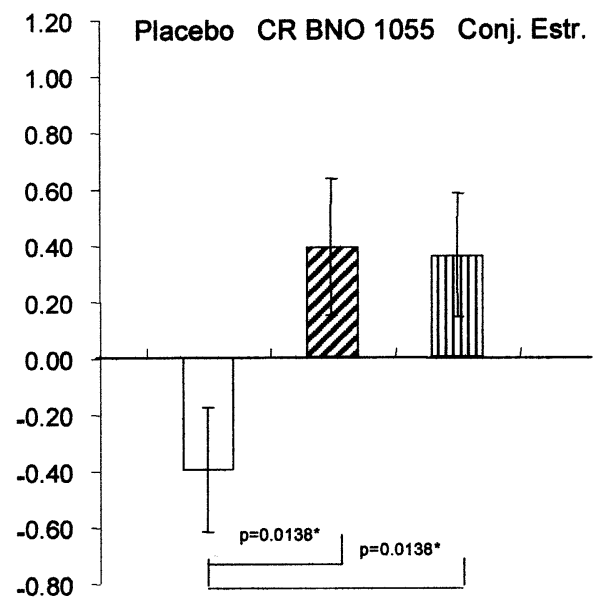

Means adjusted for baseline and center differences +/- SEM

Fig. 8. The bone turnover index (log bone-specific alkaline phosphatase: CrossLaps) was significantly increased after 12 weeks of treatment with C. racemosa BNO 1055 and the CE. Because the quotient obviously decreased in the placebo-treated women, a statistical significant improve of bone metabolism can be postulated under CR BNO 1055 and the $\mathrm{CE}$; both compounds being identically active.

eous/ethanolic C. racemosa extract BNO 1055 (see Wuttke et al., this volume). These data add to the evidence that the C. racemosa extract BNO 1055 contains yet unidentified compounds with an estrogenic effect on the hypothalamic GnRH pulse generator.

The other climacteric complaints, particularly those subsumed under factor 2 "psyche", are certainly estrogenic effects in suprahypothalamic brain structures. Most likely they are exerted in the mesolimbic systems, which express $\mathrm{ER} \alpha$ and $\mathrm{ER} \beta$ genes [28,29]. Factor 3 "atrophy" is a somatic factor and comprises vaginal dryness and rheumatic-like complaints. Vaginal dryness is clearly reduced by conventional hormone replacement therapy. In this study, an estrogenic effect was also observed under CR BNO 1055. The MRS item 10 "joint and muscle symptoms", individually evaluated, was significantly improved by both, CE and CR BNO 1055, indicating estrogenic activities of CR BNO 1055 (data not shown).

The data concerning bone metabolism deserve some detailed discussion. It is known that osteoblasts and osteoclasts are estrogen-receptive [3032]. In ovary-intact or estrogen-substituted women, the activity of osteoblasts and osteoclasts is well balanced, i.e. bone resorption initiated by osteoclasts is immediately counteracted by bone formation initiated by osteoblasts. Withdrawal of estrogen, in animal experiments, results in increased activities of both, osteoblasts and osteoclasts, i.e. an increased bone turnover [33]. Though both parameters were stimulated, the activity of osteoclasts was outbalanced, which lead to constant reduction of bone. This catabolic effect is counteracted by estrogens. Patients treated with $C$. racemosa BNO 1055 showed significantly increased serum levels of bone-specific alkaline phosphatase, which is indicative for an effect of osteoblast activity. This effect was not seen under treatment with CE. Patients treated with CE had decreased levels of serum CrossLaps, the metabolic products of bone-specific collagen- $1 \alpha 1$. Low CrossLaps levels are the indication for a decreased activity of osteoclasts. As a net effect, in bones, the results for CR BNO 1055 and CE are comparable. The ratio of the bone-specific alkaline phosphatase:CrossLaps is significantly increased under both treatments, in comparison to placebo. Although the mechanism of action may be different from that of CE, which decrease osteoclast activity, CR BNO 1055 appears to have osteoprotective effects in bones by increasing osteoblast activity.

In summary, these results clearly indicate that C. racemosa BNO 1055 (Klimadynon ${ }^{\circledR} /$ Menofem ${ }^{\circledR}$ ) has SERM activities. In postmenopausal women, CR BNO 1055 exerted the same effects as CE. Most of the climacteric complaints were significantly improved. In bones, beneficial effects on bone turnover were apparent. In contrast to CE, CR BNO 1055 exerted no effects on the endometrium but had a mild estrogenic activity in the vagina. These data highlight earlier results, obtained in rats, where similar effects for CR extract BNO 1055 were reported. Therefore, we propose that CR BNO 1055 contains substances 
with SERM activities, i.e. with desired effects in the hypothalamus, in the mesolimbic brain regions, in bones and on vaginal epithelium, but without estrogenic effects in the uterus.

\section{Acknowledgements}

This work was in part funded by the EU program "(E)UROESTROGEN(E)S" as part of the network EUROSTRERONE (Grant No. QLK6-CT-2000-00565) and by the German Research Society (Grant No. Wu 60/12-1).

\section{References}

[1] Liehr JG. Genotoxicity of the steroidal oestrogens oestron and oestradiol: possible mechanism of uterine and mammary cancer development. Hum Reprod Update 2001;7:273-81.

[2] Colditz GA, Hankinson SE, Hunter DJ, et al. The use of estrogens and progestins and the risk of breast cancer in postmenopausal women. N Engl J Med 1995;332:1589-93.

[3] Rossouw JE, Anderson GL, Prentice RL, et al. Risks and benefits of estrogen plus progestin in healthy postmenopausal women principal results from the women's health initiative randomized controlled trial. J Am Med Assoc 2002;288:321-33.

[4] Southcott BM. Carcinoma of the endometrium. Drugs 2001;61:1395-405.

[5] Feeley KM, Wells M. Hormone replacement therapy and the endometrium. J Clin Pathol 2001;54:435-40.

[6] Grodstein F, Manson JE, Colditz GA, Willett WC, Speizer FE, Stampfer MJ. A prospective, observational study of postmenopausal hormone therapy and primary prevention of cardiovascular disease. Ann Intern Med 2000;133:93341.

[7] Mikkola TS, Clarkson TB. Estrogen replacement therapy, atherosclerosis, and vascular function. Cardiovasc Res 2002;53:605-19.

[8] Hulley S, Grady D, Bush T, Furberg C, Herrington D, Riggs B, Vittinghoff E. Randomized trial of estrogen plus progestin for secondary prevention of coronary heart disease in postmenopausal women. Heart and Estrogen/ Progestin Replacement Study (HERS) Research Group, J. Am. Med. Assoc. 1998;280:605-13.

[9] Herrington DM, Potvin Klein K. Cardiovascular trials of estrogen replacement therapy. Ann NY Acad Sci 2001;949:153-62.

[10] Ariyo AA, Villablanca AC. Estrogens and lipids: can HRT designer estrogens, and phytoestrogens reduce cardiovascular risk markers after menopause? Postgrad Med 2002;111:23-30.
[11] Clarkson TB. Soy, soy phytoestrogens and cardiovascular disease. J Nutr 2002;132:566S-9S.

[12] Bjarnason NH, Hassager C, Christiansen C. Postmenopausal bone remodelling and hormone replacement. Climacteric 1998;1:72-9.

[13] Santen RJ, Pinkerton JV, Conaway M, Ropka M, Wisniewski L, Demers L, Klein KO. Treatment of urogenital atrophy with low-dose estradiol: preliminary results. Menopause 2002;9:179-87.

[14] Einer-Jensen N, Zhao J, Andersen KP, Kristoffersen K. Cimicifuga and Melbrosia lack oestrogenic effects in mice and rats. Maturitas 1996;25:149-53.

[15] Seidlová-Wuttke D, Jarry H, Viereck V, Heiden I, Christoffel V, Spengler B, Wuttke W. Cimicifuga racemosa: a selective estrogen receptor modulator? In: Samsioe G, Skouby S, editors. Midlife Health - Current Concepts and Challenges for the Future. Amsterdam: Elsevier, 2001.

[16] Liske E, Hänggi W, Henneicke-vonZepelin HH, Boblitz N, Wüstenberg P, Rahlfs VW. Physiological investigation of a unique extract of black cohosh (Cimicifugae racemosae rhizoma): a 6-month clinical study demonstrates no systemic estrogenic effect. J Womens Health Gend Based Med 2002;11:163-74.

[17] Gajdos C, Jordan VC. Selective estrogen receptor modulators as a new therapeutic drug group: concept to reality in a decade. Clin Breast Cancer 2002;2:272-81.

[18] Anderson PW, Cox DA, Sashegyi A, Paul S, Silfen SL, Walsh BW. Effects of raloxifene and hormone replacement therapy on markers of serum atherogenicity in healthy postmenopausal women. Maturitas 2001;25:71-7.

[19] Note for guidance on good clinical practice, CPMP/ICH/ 135/95. International Conference on Harmonisation of Technical Requirements for Registration of Pharmaceuticals for Human Use (ICH), London: Committee for Proprietary Medicinal Product (CPMP), 1997.

[20] World Medical Association Declaration of Helsinki. Ethical principles for Medical Research Involving Human Subjects, last amended by the 48th World Medical Assembly, Somerset West, Republic of South Africa, 1996.

[21] Dallal GE. Design-Power Analysis and Expected Mean Squares. A Supplementary Module for SYSTAT and SYGRAPH. Evanston, IL: Systat, Inc, 1988.

[22] Hauser GA, Huber IC, Keller PJ, Lauritzen C, Schneider HPG. Evaluation der klimakterischen beschwerden (menopause rating scale (MRS)) zentralblatt für gynäkologie 1994;116:16-23.

[23] Schneider HPG, Rosemeier HP, Schnitker J, Gerbsch S, Turck R. Application and factor analysis of the menopause rating scale (MRS) in a post-marketing surveillance study of Climen. Maturitas 2000;37:113-24.

[24] Kuhl H, Taubert H-D. Das Klimakterium, Pathophysiologie-Klinik-Therapie. Stuttgart, NY: Georg Thieme, 1987:178-81.

[25] Stoll W. Phytotherapeutikum beeinflusst atrophisches vaginalepithel: doppelblindversuch Cimicifuga vs Östrogenpräparat (phytopharmacon influences atrophic vaginal 
epithelium: double-blind study - Cimicifuga vs. estrogenic substances). Therapeuticum 1987;1:23-31.

[26] Tataryn IV, Lomax P, Bajorek JG, et al. Postmenopausal hot flushes: a disorder of thermoregulation. Maturitas 1980;2:101-7.

[27] Jarry H, Perschl A, Wuttke W. Further evidence that preoptic anterior hypothalamic GABAergic neurons are part of the GnRH pulse and surge generator. Acta Endocrinol 1988;118:573-9.

[28] Shughrue PJ, Lane MV, Merchenthaler I. Comparative distribution of estrogen receptor-alpha and -beta mRNA in the rat central nervous system. J Comp Neurol 1997;388:507-25.

[29] Shughrue PJ, Merchenthaler I. Distribution of estrogen receptor beta immunoreactivity in the rat central nervous system. J Comp Neurol 2001;436:6481 .
[30] Bord S, Horner A, Beavan S, Compston J. Estrogen receptors alpha and beta are differentially expressed in developing human bone. J Clin Endocrinol Metab 2001;86:2309-14.

[31] Okazaki R, Inoue D, Shibata M, et al. Estrogen promotes early osteoblast differentiation and inhibits adipocyte differentiation in mouse bone marrow stromal cell lines that express estrogen receptor (ER) alpha or beta. Endocrinology 2002;143:2349-56.

[32] Braidman IP, Hainey L, Batra G, Selby PL, Saunders PT, Hoyland JA. Localization of estrogen receptor beta protein expression in adult human bone. $\mathrm{J}$ Bone Miner Res 2001;16:214-20.

[33] Westerlind KC, Wronski TJ, Ritman EL, et al. Estrogen regulates the rate of bone turnover but bone balance in ovariectomized rats is modulated by prevailing mechanical strain. Proc Natl Acad Sci USA 1997;94:4199-204. 